\title{
Selective gray and texture information for image segmentation
}

\author{
Kaiqiong $\operatorname{Sun}^{1}$ \\ ${ }^{1}$ School of Mathematics and Computer Science, Wuhan \\ Polytechnic University, \\ Wuhan 430023, China
}

\begin{abstract}
In this paper, an active contour model is proposed for image segmentation, combining original image and feature image information with adaptive weight. The feature image is a texture descriptor which intrinsically defines the geometry of textures using semi-local image information. The Kullback-Leibler distance is used to design the energy of active contour model, in which the weight coefficient for original image and feature image is decided by the ratio of their distributions entropy. The energy minimization is achieved with Split-Bregman method. The results show that proposed method can achieve automatic segmentation of natural image with minimal parameter compared to related approaches.
\end{abstract}

Keywords: active contour model; Kullback-Leibler distance; Split-Bregman method

\section{Introduction}

Image segmentation is an essential part of the image processing and computer vision. Among different segmentation methods, active contour model is a widely adopted framework. However, the energy functional of popular active contour model is nonconvex, which leads to the segmentation result being prone to local minima and can not achieve the desired segmentation. In view of this problem, Bresson et al. [3] proposed the fast global minimization algorithm of active contour models. In above method, the active contours without edges [2] model and the geodesic active contours [1] model are combined into the global minimum model, and both the image edge and intensity region can be detected. The dual algorithm based on global variational norm is introduced in the algorithm, which avoids the shortcoming of the time consuming of the level set method and the repeated reinitialization

The popular edge and region based active contours [1], [2] depend on gray information and can not efficiently deal with texture image or image with complex background. Under the framework of global minimization algorithm model in [3], $\mathrm{Ni}$ et al. [4] used the local histogram information in the active contour model, and adopted Wasserstein distance to determine the dissimilarity between two histograms. The Wasserstein distance is a metric and is

\author{
Zhiqin Wan ${ }^{2}$ \\ ${ }^{2}$ School of Measuring and Optical Engineering, Nanchang \\ Hangkong University \\ Nanchang 330063, China
}

able to faithfully measure the distance between two histograms, compared to many pointwise distances. Therefore, it is insensitive to oscillations, and robust to noise. The model can segment image with texture and complex background. However, the local histogram information for texture depends on the size of scale used in local histogram computation.

In [5], an unsupervised segmentation method based on the Kullback-Leibler distance and on non-parametric estimation of the probability distribution function was introduced and solved with the fast minimization scheme defined in [3]. More recently, a new technique based on the Split-Bregman method [6] has been introduced to solve the convexified active contour model in a faster way than [3]. A texture descriptor based on differential geometry metric was used for texture segmentation in the fast active contour model proposed by Houhou et al. [7]. In the model, the popular Kullback-Leibler distance is used to design the energy of active contour model which distinguishes the background and textures of interest. The Split-Bregman method [6] is adopted to minimization of energy functional. This method is efficient for the texture image segmentation. However, the model is also depending on the size of scale used for texture descriptor computation.

The texture descriptor in [7] is computed from the nonlocal image information. Thus, the method is only proper for texture image segmentation. When dealing with images of different gray property, it lacks an adaptive selection or switch between local and nonlocal image information.

\section{Related work}

To overcome the inefficiency of general region based active that uses gray information in texture segmentation, a fast texture segmentation based on semi-local region descriptor and active contour was proposed in [7].

In the model [7], nonlocal image information $\varphi_{\mathrm{xy}}$ is used, which is the square patch of size $\tau \times \tau$ around the pixel as,

$$
\varphi_{x, y}(I)=\left\{I\left(x+t_{x}, y+t_{y}\right)\right\}, \quad t_{x}, t_{y} \in\left[-\frac{\tau}{2}, \frac{\tau}{2}\right]
$$

where $I$ is the original image. Based on above gray information, a geometry metric $g_{x y}$ is defined as, 


$$
g_{x y}=\left(\begin{array}{ll}
1+\left(\partial_{x} \varphi_{x, y}\right)^{2} & \partial_{x} \varphi_{x, y} \partial_{y} \varphi_{x, y} \\
\partial_{x} \varphi_{x, y} \partial_{y} \varphi_{x, y} & 1+\left(\partial_{y} \varphi_{x, y}\right)^{2}
\end{array}\right)
$$

A feature image $F$ is then formulated with the geometry metric as following,

$$
F=\exp \left(-\frac{\operatorname{det}\left(g_{x y}\right)}{\sigma^{2}}\right)
$$

where $\sigma$ is a scale. For any curve, the inside and outside probability density functions of the feature image can be calculated as,

$$
\begin{aligned}
& q_{\text {in }}(F, \Omega)=\frac{1}{|\Omega|} \int_{\Omega} K(F-F(\hat{x})) d \hat{x} \\
& q_{\text {out }}(F, \Omega)=\frac{1}{\left|\Omega_{0} \backslash \Omega\right|} \int_{\Omega_{0} \backslash \Omega} K(F-F(\hat{x})) d \hat{x}
\end{aligned}
$$

where $\Omega_{0}$ denotes the image domain and $\Omega$ denotes the inner region of curve. $K$ is a Gaussian kernel. The Kullback-Leibler $(K L)$ distance between the two distributions is defined as below,

$$
\begin{aligned}
K L(F, \Omega) & =\int_{R_{+}}\left(q_{\text {in }}(F, \Omega) \log \frac{q_{\text {in }}(F, \Omega)}{q_{\text {out }}(F, \Omega)}\right. \\
& \left.+q_{\text {out }}(F, \Omega) \log \frac{q_{\text {out }}(F, \Omega)}{q_{\text {in }}(F, \Omega)}\right) d F
\end{aligned}
$$

The energy of active contour is formulated as,

$$
E(F, \Omega)=-\lambda \cdot K L(F, \Omega)+\int_{\Omega \Omega} d s
$$

Thus the objective of the energy minimization is to maximize the $K L$ distance while maintaining normalization of curve. The minimization of the energy functional is implemented with Split-Bregman method [6].

\section{Proposed method}

The texture descriptor defined in last section is proper for texture but it cannot distinguish the region defined by local image information which traditional model [2] incorporates. To combine the local and nonlocal image information, we propose the following energy

$$
\begin{aligned}
E(I, F, \Omega)= & -\lambda\{\alpha \cdot K L(I, \Omega)+\beta \cdot K L(F, \Omega)\} \\
& +\int_{\Omega \Omega} d s
\end{aligned}
$$

Where $I$ is the original image, and $F$ is the feature image defined in last section. $\alpha, \beta$ and $\lambda$ are positive constants. $K L(I, \Omega)$ and $K L(F, \Omega)$ are the $K L$ distances between inner and outer region of curve defined on original image and feature image, respectively. When $\alpha=0$, the energy define in (2) equal to that in (1).

To make the model selectively choose gray or texture information for segmentation, adaptive weight coefficients $\alpha, \beta$ for original image and feature image are decided by a sigmoid function of the entropy of distribution as follows,

$$
\alpha / \beta=1-1 /\left(1+\exp \left(-\left(E_{I / F}-E_{\text {mean }}\right)\right)\right)
$$

where $E_{\mathrm{I}}$ and $E_{\mathrm{F}}$ are the entropy of distribution in the original image and feature image, respectively.
$E_{\text {mean }}=\left(E_{\mathrm{I}}+E_{\mathrm{F}}\right) / 2$. Above function produces big weight parameter for the distribution with small entropy. The SplitBragman [6] iterative scheme is used to minimize (2).

\section{Experiments}

Several images have been used to test the proposed method and comparative results to other method are presented.

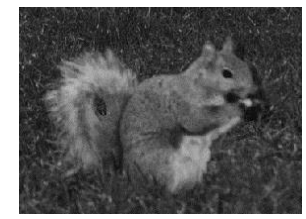

(a)

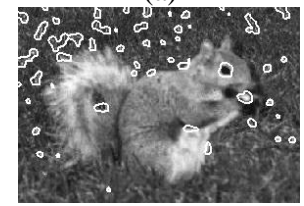

(c)

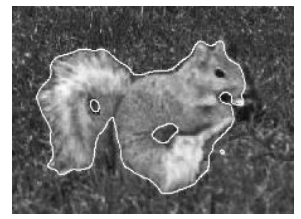

(b)

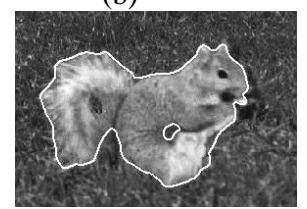

(d)
Fig. 1: Original image and segmentation results with different methods. (a) original image, (b) segmentation result with global region information [2], (c) segmentation result with the texture feature information [7], (d) segmentation result with selective gray and texture information by proposed method.

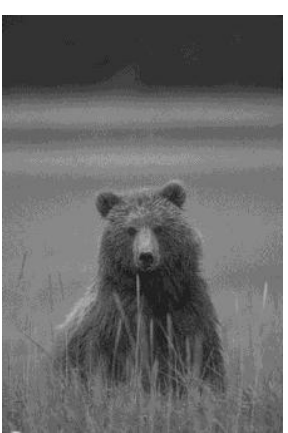

(a)

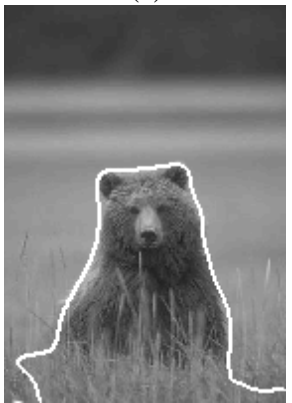

(c)

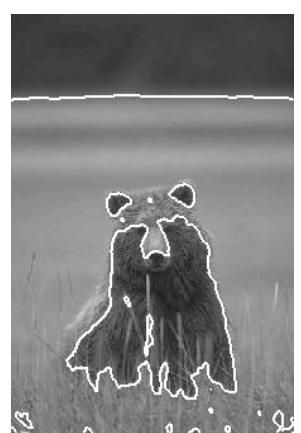

(b)

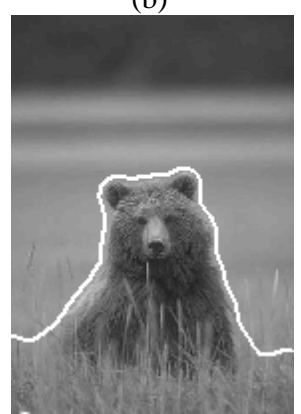

(d)
Fig. 2: Original image and segmentation results with different methods. (a) original image, (b) segmentation result with global region information [2], (c) segmentation result with the texture feature information [7], (d) segmentation result with selective gray and texture information by proposed method. 
Fig. 1(a) is an original image. The object and background can be distinguished with original gray information. Fig. 1(b) shows the segmentation result with global region information by [2]. On the feature image obtained by previously mentioned method, the distributions of object and background overlaps strongly. Fig. 1(c) shows the result by minimizing the energy in (1). Fig. 1(d) shows the result by proposed method where a automatic selection of gray and texture image was obtained. Accordingly, Fig. 2 shows the results on another image.

\section{Conclusion}

A wide range of image feature have been proposed for segmentation of image with different properties. Normally, one kind of image feature fits image with special property. Even in the case of proper image feature for segmentation, there is also a problem of selecting optimal parameter of image feature, such as the local scale in [4] and patch size in [7]. In order to make the segmentation method have adaptability for image with different property, multiple image feature and more importantly a combination strategy for them is needed. In this paper, the original image and texture feature image information are combined with adaptive weight in the active contour segmentation. The weight coefficient for original image and feature image is decided by the ratio of their distributions entropy. The combination of different image information and the adaptive weight coefficient makes the method is proper for segmentation of broader range of image in a unified formulation.

\section{Acknowledgement:}

Supported by the National Natural Science Foundation of China under Grant 61163046 and the Natural Science Foundation of Hubei Province of China under Grant 2014 CFB881.

\section{References}

[1] V. Caselles, R. Kimmel, G. Sapiro, “Geodesic active contours," Int. J. Comput.Vis., vol. 22, pp. 61-79. 1997.

[2] T.F. Chan, L. Vese, "Active contours without edges," IEEE Trans. Image Process., vol. 10, pp. 266-277, 2001.

[3] X. Bresson, S. Esedoglu, P. Vandergheynst, et al., "Fast global minimization of the active contour/snake model," Journal of Mathematical Imaging and Vision, vol. 28, pp. 151-167, 2007.

[4] K. Ni, X. Bresson, T. Chan, S. Esedoglu. "Local Histogram based Segmentation Using the Wasserstein Distance," Int. J. Computer. Vis., vol. 84, pp. 97-111, 2009.

[5] N. Houhou, J-P. Thiran, and X. Bresson, "Fast Texture Segmentation Model based on the Shape Operator and Active Contour," In Computer Vision and Pattern Recognition, 2008.

[6] T. Goldstein, X. Bresson, and S. Osher, "Geometric Applications of the Split Bregman Method: Segmentation and Surface Reconstruction," CAM Report 09-06, 2009.

[7] N. Houhou, J. P. Thiran, X. Bresson, "Fast texture segmentation based on semi-local region descriptor and active contour," Numerical Mathematics: Theory, Methods and Applications, vol. 2(EPFL-ARTICLE140431), pp. 445-468, 2009. 